\title{
Teriflunomide for the treatment of relapsing- remitting multiple sclerosis: patient preference and adherence
}

This article was published in the following Dove Press journal:

Patient Preference and Adherence

9 February 2015

Number of times this article has been viewed

\author{
Antonios Bayas' \\ Mathias Mäurer² \\ 'Department of Neurology, Klinikum \\ Augsburg, Augsburg, Germany; \\ 2Department of Neurology, Caritas \\ Krankenhaus Bad Mergentheim, \\ Bad Mergentheim, Germany
}

\begin{abstract}
Multiple sclerosis (MS), a chronic demyelinating neuroinflammatory disease of the central nervous system, is the most common neurological disorder leading to disability in young adulthood. In the last 2 decades, numerous treatments for relapsing-remitting MS have been approved with eleven treatment options available worldwide. One of the determinants in treatment selection is disease activity in the individual patient. However, patient preferences play an increasingly major role in treatment decision making. With teriflunomide, a reversible inhibitor of the enzyme dihydroorotate dehydrogenase, a new oral therapeutic option, given once daily, has been approved within the last 2 years by the regulatory agencies. The current review focuses on characteristics of the drug relevant for patients' preferences in the treatment decision process in the light of the available medications. Perceiving and considering patients' preferences will have an effect on treatment adherence, which is known to be often low in MS patients. Teriflunomide-related adherence issues will also be discussed regarding mode of application, dosing, and potential side effects.
\end{abstract}

Keywords: disease modifying treatment, oral, first line, pharmacovigilance

\section{Introduction}

Multiple sclerosis (MS) is a chronic demyelinating neuroinflammatory disease of the central nervous system, affecting approximately 2 million people worldwide. The etiology is assumed to be multifactorial involving, for example, environment and genetic predisposition, affecting disease development. MS is the most common neurological disorder leading to disability in young adulthood. Clinical disease starts with recurrent and often reversible neurological deficits in early adulthood in the majority of patients. MS can follow three different clinical courses: relapsing-remitting (RR), secondary progressive, and primary progressive MS.

In the past 2 decades, numerous immunoprophylactic and, to a much lesser extent, symptomatic treatments have been approved for MS, especially for RR MS. The disease cannot be cured by the available treatments so far, but a reduction of relapse rate, disease progression and other clinical activity markers, eg, magnetic resonance imaging (MRI) lesions and brain atrophy, could be achieved.

\section{Treatment options in MS}

Treatment of relapses as well as immunoprophylactic and symptomatic treatment are the mainstays of modern MS therapy.

For many years, interferon beta preparations and glatiramer acetate (Table 1) have

belonged to the well-established group of injectable immunoprophylactic therapeutics,
Department of Neurology, Klinikum

Augsburg, Stenglinstr 2, 86156

Augsburg, Germany

Tel +4982I 4003892

Fax +49 821 400 2691

Email antonios.bayas@klinikum-augsburg.de

submit your manuscript $\mid$ www.dovepress.con 
Table I Treatment options for RR MS (in alphabetical order)

\begin{tabular}{|c|c|}
\hline Treatment & Dosing frequency \\
\hline \multicolumn{2}{|l|}{ Infusions } \\
\hline Alemtuzumab (Lemtrada ${ }^{\circledR}$; Genzyme Therapeutics Ltd, Oxford, UK) & $\begin{array}{l}12 \text { mg; first year: } 5 \text { infusions on consecutive days; } \\
\text { second year: } 3 \text { infusions on consecutive days }\end{array}$ \\
\hline Mitoxantrone (Ralenova ${ }^{\circledR}$; MEDA Pharma GmbH \& Co. KG, Bad Homburg, Germany) & $\begin{array}{l}12 \mathrm{mg} / \mathrm{m}^{2} \text { body surface every } 3 \text { months (maximal } \\
\text { cumulative dose } 140 \mathrm{mg} / \mathrm{m}^{2} \text { ) }\end{array}$ \\
\hline Natalizumab (Tysabri ${ }^{\circledR}$; Biogen Idec Limited, Maidenhead, Berkshire, UK) & 300 mg every 4 weeks \\
\hline \multicolumn{2}{|l|}{ Injectables } \\
\hline Glatiramer acetate sc (Copaxone ${ }^{\circledR}$; TEVA Pharmaceuticals USA, Inc., North Wales, PA, USA) & Once daily $(20 \mathrm{mg})$ or tiw $(40 \mathrm{mg})^{*}$ \\
\hline Interferon beta-Ia im (Avonex ${ }^{\circledR}$; Biogen Idec Limited) & Weekly $30 \mu \mathrm{g}$ \\
\hline Interferon beta-Ia sc (Rebif ${ }^{\circledR}$; Merck Serono Europe Limited, London, UK) & tiw 22 or $44 \mu g$ \\
\hline Interferon beta-Ia sc (Plegridy ${ }^{\circledR}$; Biogen Idec Limited) & Every 2 weeks $125 \mu \mathrm{g}$ \\
\hline Interferon beta-Ib sc (Betaferon ${ }^{\circledR}$; Bayer AG, Leverkusen, Germany; and Extavia ${ }^{\circledR}$; Novartis & Every other day $250 \mu \mathrm{g}$ \\
\hline \multicolumn{2}{|l|}{ Europharm Limited, West Sussex, UK) } \\
\hline \multicolumn{2}{|l|}{ Oral } \\
\hline Dimethyl fumarate (Tecfidera ${ }^{\circledR}$; Biogen Idec Limited) & bid $240 \mathrm{mg}$ \\
\hline Fingolimod (Gilenya ${ }^{\circledR}$; Novartis Europharm Limited, West Sussex, UK) & Once daily $0.5 \mathrm{mg}$ \\
\hline Teriflunomide (Aubagio ${ }^{\circledR}$; Sanofi-Aventis, Paris, France) & Once daily $7 \mathrm{mg}$ or 14 mg* \\
\hline
\end{tabular}

Note: *Approval and dosing dependent on national regulations.

Abbreviations: bid, twice daily; im, intramuscular; MS, multiple sclerosis; RR, relapsing-remitting; sc, subcutaneous; tiw, three times weekly.

with a well-known safety profile for use in RR and (interferon beta- $1 \mathrm{~b}$ subcutaneously [sc], interferon beta-1a sc three times per week [tiw]), secondary progressive MS with inflammatory disease activity, which is characterized by relapses. Interferon beta- $1 \mathrm{~b}$ was licensed as the first beta interferon in the US in 1993, and glatiramer acetate in 1996. For MS patients with highly active disease, natalizumab and mitoxantrone have been introduced as further treatment options. In 2010, fingolimod, a sphingosine phosphate receptor agonist, was licensed by the US Food and Drug Administration (FDA) as the first modern oral immunoprophylactic treatment. In most European countries, fingolimod is restricted to patients with high clinical and MRI disease activity.

Within the last 2 years in the US and European Union, two further oral drugs have been approved: dimethyl fumarate (DMF), given as capsules twice daily (bid); and teriflunomide with a once-daily application. Alemtuzumab was licensed by the European Medicines Agency in 2013 for RR MS, and the FDA approved alemtuzumab in autumn 2014.

In the growing field of currently available disease modifying treatments (DMTs), it is crucial to identify factors for patients' treatment decisions. The current review focuses on teriflunomide as a new treatment option in RR MS, in particular considering patients' preference and adherence.

PubMed was searched using the terms "teriflunomide AND multiple sclerosis", "teriflunomide AND adherence", "teriflunomide AND preference", "multiple sclerosis AND adherence", and "multiple sclerosis AND preference" without time-period restriction. The references of the resulting studies were used to identify additional articles to be included in the review. Additionally, congress abstracts presented in 2014 have been selected.

\section{Teriflunomide - efficacy and safety}

Teriflunomide is an immunomodulatory drug with antiinflammatory properties. In 2012, the FDA approved Teriflunomide $7 \mathrm{mg}$ and $14 \mathrm{mg}$ once daily for the treatment of adult patients with RR MS. In 2013, the $14 \mathrm{mg}$ dose was approved for the treatment of RR MS patients by the European Medicines Agency.

Teriflunomide reversibly inhibits the enzyme dihydroorotate dehydrogenase - a key enzyme for the de novo synthesis of pyrimidine - thus leading to a reduced proliferation of autoreactive T- and B-cell clones. Resting and slowly proliferating cells can fulfil their demand from the existing pyrimidine pool and remain unaffected. ${ }^{1}$

After oral intake, teriflunomide is rapidly absorbed; the bioavailability is almost $100 \%$. More than $99 \%$ of the active substance is bound to plasma proteins. There is almost no involvement of the CYP450 system, which may decrease potentially clinically relevant drug-drug interactions. Nevertheless, some interactions have to be kept in mind (eg, with warfarin; for details see summary of product characteristics ${ }^{2}$ ). Teriflunomide is almost exclusively excreted via the feces and undergoes extensive enterohepatic recycling. The halflife is approximately 19 days. To achieve a rapid decrease in the plasma level, the oral administration of cholestyramine is required (dosage: $8 \mathrm{~g}$ three times daily (tid) for 11 days, 
or $4 \mathrm{~g}$ tid when cholestyramine $8 \mathrm{mg}$ is not tolerated). Alternatively, the administration of activated charcoal is possible ( $50 \mathrm{~g}$ charcoal powder, administered every 12 hours over a period of 11 days). ${ }^{3}$

Teriflunomide has been studied in an extensive clinical development program including two placebo-controlled Phase III studies: TEMSO (Teriflunomide Multiple Sclerosis Oral) and TOWER (Teriflunomide Oral in people With relapsing multiplE scleRosis).

TEMSO is a placebo-controlled, double-blind trial that included 1,086 patients with RR MS from 126 centers in 21 countries. ${ }^{4}$ The patients were between 18 and 55 years old and had a mean Expanded Disability Status Scale of 2.7. They were required to have an active disease defined by at least one exacerbation in the previous 12 months or two relapses in the previous 24 months. About one-quarter of the patients were pretreated with immunomodulatory agents.

During the 2-year study period, patients received either placebo, teriflunomide $7 \mathrm{mg}$ or $14 \mathrm{mg}$ once daily. The primary endpoint was the annualized relapse rate. Secondary endpoints included disability progression and several MRI parameters (number of T1 gadolinium-enhanced lesions, T2 lesion load defined by new and enlarging T2 lesions). Teriflunomide $14 \mathrm{mg}$ significantly reduced the annualized relapse rate by $31 \%$ compared to placebo $(P<0.001)$. In addition, $14 \mathrm{mg}$ teriflunomide resulted in a significant risk reduction of disability progression of $30 \%(P=0.02)$ compared to placebo. The analysis of the MRI data showed a significantly reduced number of gadolinium-enhancing $\mathrm{T} 1$ lesions and of new and enlarging T2 lesions (69\%) for the $14 \mathrm{mg}$ dose. A significant reduction of the annualized relapse rate was also observed for the $7 \mathrm{mg}$ dose; however, the response of the other parameters (in particular MRI) was clearly dose dependent with superiority of the $14 \mathrm{mg}$ dose.

The international, multicenter, double-blind, placebo-controlled Phase III study TOWER included 1,169 patients with RR MS from 26 nations. Patients were 18-55 years old and had experienced at least one exacerbation in the past year, or two relapses in the last 2 years. The minimum duration of treatment was 48 weeks; the average time on treatment was 18 months. ${ }^{5}$

The study participants received either placebo or teriflunomide $7 \mathrm{mg}$ or $14 \mathrm{mg}$ once daily. Again, the primary endpoint was the annualized relapse rate. Progression of disability confirmed after 12 weeks was one of the secondary endpoints. The significant reduction of the annualized relapse rate in patients treated with $14 \mathrm{mg}$ teriflunomide could be reproduced in the TOWER study. Administration of $14 \mathrm{mg}$ teriflunomide resulted in a relative risk reduction of $36.3 \%(P<0.0001)$ compared to placebo. In addition, more patients remained relapse-free during the entire study period $(P<0.0001)$. Finally, the risk of disability progression was reduced significantly by $31.5 \%(P=0.0442)$. Thus, teriflunomide is the only oral treatment option that has shown a significant reduction in disability progression in two pivotal studies.

In TEMSO, an overall good tolerability of teriflunomide could be observed. The incidence of adverse events was comparable in all study groups, including the placebo group. An increase in liver enzymes, diarrhea, nausea, and decreased hair density was more frequently observed in patients treated with teriflunomide compared with placebo. Discontinuation rates showed also no significant differences between the treatment arms. There was no increased risk for opportunistic infections and no increased risk for serious infections in patients treated with teriflunomide. Furthermore, no increased risk of malignancy was observed. Fortunately, no deaths occurred during the study. This good tolerability and favorable side effect profile was also observed in the TEMSO extension study.

In the TOWER study, the favorable safety profile was reproduced; no unexpected safety signals were observed. The infection rate was similar between the treatment arms, and there was also no evidence of an increased rate of malignant tumors. The most common side effects were mild elevation of liver enzymes, a slight increase in blood pressure, and a decreased hair density. In the TOWER study, three deaths (one traffic accident, one suicide, and one case of sepsis) occurred in the teriflunomide arms. All death were unrelated to the study treatment. In the placebo arm, a death occurred as a result of a respiratory infection.

In order to evaluate the clinical efficacy of teriflunomide in comparison to well-established immunomodulatory drugs, the efficacy of 48-115 (median 63.6) weeks' treatment with oral teriflunomide 7 and $14 \mathrm{mg}$ /day versus subcutaneous interferon beta-1a 44 tiw was investigated in a randomized, single-blind, multicenter, Phase III study (TENERE; n=324) in patients with RR MS. The participants were aged 18 years or older and had an Expanded Disability Status Scale $\leq 5.5{ }^{6}$

The core treatment period lasted until 48 weeks after randomization of the last patient. Teriflunomide recipients were blinded as to dosage; interferon beta-1a treatment was open-label. The primary endpoint was the time to treatment failure in the intent-to-treat population during the core treatment period. Treatment failure was defined as the occurrence of a disease relapse or study discontinuation for any reason. 
The secondary outcome measures included annualized relapse rate, patient-reported fatigue using the Fatigue Impact Scale, and patient satisfaction assessed with the Treatment Satisfaction Questionnaire for Medication.

In patients receiving $14 \mathrm{mg}$ teriflunomide ( $\mathrm{n}=111), 37.8 \%$ reached the primary endpoint, while treatment failure was observed in $42.3 \%$ of patients treated with interferon beta-1a $(n=104)$. This difference was statistically not significant. There was also no statistically significant difference between teriflunomide $14 \mathrm{mg}$ and interferon beta in the adjusted annualized relapse rate.

However, treatment satisfaction of patients treated with teriflunomide was significantly higher than in patients treated with interferon beta-1a. This result was based on the clearly superior ranking in the subscales "side effects" and "ease of therapy", while no differences were observed in the subscale "clinical efficacy" (Figure 1).

Finally the efficacy and safety of teriflunomide in patients with a first clinical episode suggestive of MS were evaluated (TOPIC study). Six hundred and eighteen patients were enrolled and randomly assigned to teriflunomide $14 \mathrm{mg}$ $(n=216)$, teriflunomide $7 \mathrm{mg}(n=205)$, or placebo $(n=197)$. Compared with placebo, teriflunomide significantly reduced the risk of relapse defining clinically definite MS (new neurological abnormality separated by at least 30 days from the onset of a preceding clinical event, present for at least 24 hours, and occurring in the absence of fever or known infection) at the $14 \mathrm{mg}$ dose $(42.6 \%, P=0.0087)$ and at the $7 \mathrm{mg}$ dose $(37.2 \%, P=0.0271) .^{7}$ Interpretation of the findings was limited because of early cessation of the study,

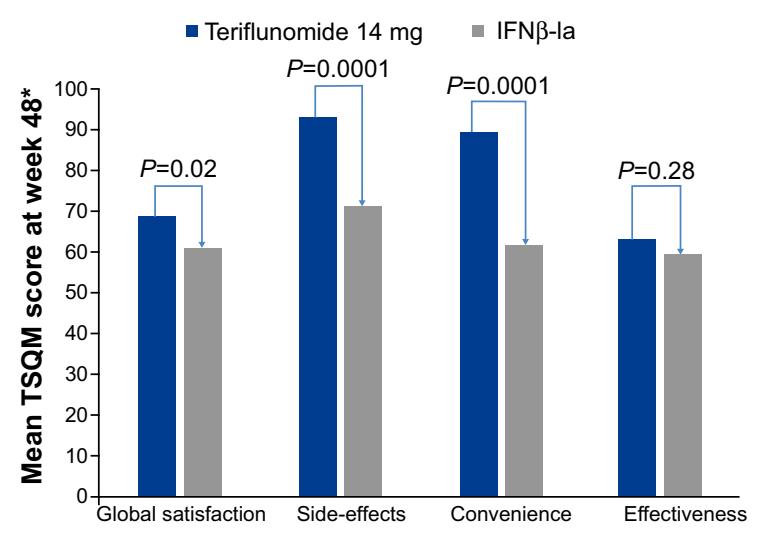

Figure I Treatment satisfaction at week 48 was significantly improved with teriflunomide $14 \mathrm{mg}$ compared with sc IFN $\beta$-la in the TSQM domains of global satisfaction, side-effects, and convenience.

Notes: *Least square mean values. Mixed-effect model with repeated measures adjusted for Expanded Disability Status Scale strata, region, visit, and treatment-byvisit interaction. ${ }^{6}$

Abbreviations: IFN, interferon; TSQM, Treatment Satisfaction Questionnaire for Medication. reducing its statistical power. Nevertheless, the findings from the Phase III TOPIC study in clinically isolated syndrome, together with those from the TEMSO and TOWER studies in relapsing MS, support the beneficial effect of teriflunomide across a broad range of patients.

\section{Teriflunomide - guidelines for clinical practice}

Due to the reported side effects in clinical studies, safety monitoring guidelines for teriflunomide include regular monitoring of blood pressure and liver function tests (Figure 2). In addition, a complete blood count is required before initiation, and then if signs/symptoms of severe infection or hematologic toxicity occur.

The teratogenic potential of leflunomide and teriflunomide has been demonstrated in animals. ${ }^{8}$ The FDA categorizes teriflunomide into pregnancy risk category $X$. Therefore, strict contraception is recommended for all females of reproductive age. To date, there is no evidence that teriflunomide has an effect on fertility or reproductive performance. In the pivotal clinical trials, women who became pregnant while on teriflunomide were required to perform the washout procedure. A teriflunomide plasma level $<0.02 \mathrm{mg} / \mathrm{L}$ is considered as acceptable regarding safety during pregnancy. A recent analysis of the pregnancies (70 patients exposed with teriflunomide) reported in all clinical trials found no structural or functional deficits in any newborns with prenatal teriflunomide exposure. Furthermore, the proportion of spontaneous abortions was within population-based norms. ${ }^{9}$

Vaccination with inactivated vaccines is possible during treatment with teriflunomide. This was documented in a study including $128 \mathrm{MS}$ patients receiving influenza vaccine (against H1N1, H3N2, and B) while being treated with teriflunomide (TERIVA study). More than $90 \%$ of the patients showed an adequate immune response to vaccination with development of sufficiently high antibody titers. ${ }^{10}$

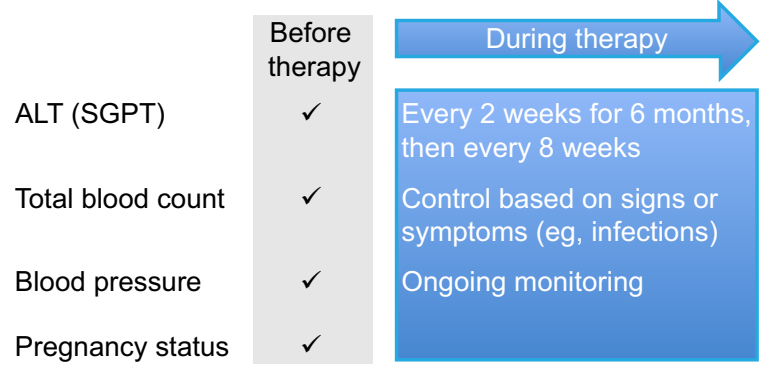

Figure 2 Safety monitoring guidelines for teriflunomide.

Abbreviations: ALT, alanine aminotransferase; SGPT, serum glutamic pyruvic transaminase. 
If a patient would like to switch from interferon beta or glatiramer acetate to teriflunomide, no washout period is required. Add-on studies combining interferon beta or glatiramer acetate with teriflunomide have raised no safety concerns. In contrast, when a switch to an escalation treatment with natalizumab, fingolimod, or alemtuzumab is necessary, a washout procedure with cholestyramine or activated charcoal is highly recommended.

\section{Patient preference for disease- modifying MS treatments - factors affecting treatment decision}

With the increasing number of available DMTs for RR MS, the decision of how to treat the individual patient has become more complex within recent years. Nowadays, there are eleven agents (one given intramuscularly, four given sc, three given intravenously, and three given orally) licensed for RR MS in the US and Europe (Table 1). For patients with a clinically isolated syndrome, when the diagnosis of MS cannot yet be established according to the revised McDonald criteria, ${ }^{11}$ only beta interferons and glatiramer acetate are approved by the regulatory agencies.

Patient counseling in the treatment decision process should focus on different aspects: disease type and activity, the benefit-risk assessment, individual contraindications, and patients' preferences.

\section{Disease activity and benefit-risk assessment}

The currently licensed treatments for RR MS differ not only by the route of administration, but also by the spectrum of risks and possible side effects. The use of natalizumab is limited due to the risk of progressive multifocal leukoencephalopathy (PML). In mitoxantrone cardiotoxicity, limiting the treatment duration, and acute myelogenous leukemia are potential side effects. For this reason, both agents are mainly reserved for patients with breakthrough disease under other DMTs. However, there is evidence to suggest the use of natalizumab or mitoxantrone as induction therapy for patients with an early aggressive disease course, characterized by frequent relapses with incomplete remission and accumulation of MRI lesions. ${ }^{12}$ Fingolimod can be used in the US without restriction regarding disease activity, whereas in most European countries it is licensed only for highly active RR MS patients. Alemtuzumab is approved for patients with active disease, defined by clinical or imaging features, in Europe, but national recommendations may restrict its use for patients with higher disease activity, relating to the inclusion criteria in the phase 3 studies. ${ }^{13}$ Beta interferons, glatiramer acetate, teriflunomide, and DMF are approved irrespective of disease activity, but are mainly used as first-line agents. When treatment decisions are made against the backdrop of the benefit-risk assessment, patients' decisions will depend on the estimate of the disease activity by the treating neurologist, but their preferences will influence the choice of treatment. It is worthwhile noting that the risk estimation of a given treatment may differ between the neurologist and the patient. Regarding the risk of PML in natalizumab treated patients, in one study, patients were willing to accept a higher risk of PML than their neurologists. ${ }^{14}$

\section{Contraindications}

The choice of treatment will also be determined by contraindications for the potential medication. According to the summary of product characteristics, they include (but are not limited to): hypersensitivity to the drugs and/or the excipients and components for all medications; certain cardiac conditions and treatment with some antiarrhythmic drugs are contraindications for the use of fingolimod; current or history of PML precludes the use of natalizumab; HIV infection precludes the use of alemtuzumab; and severe hepatic impairment contraindicates the use of fingolimod, beta interferons, and natalizumab. ${ }^{15-23}$

A contraindication for teriflunomide is severe hepatic impairment. Furthermore, it may not be given in pregnant patients or women of childbearing potential who are not using reliable contraception, since it may cause fetal harm when administered in pregnancy. Coadministration of teriflunomide with leflunomide is not allowed. DMF is not recommended in pregnancy or in women of childbearing potential who are not using appropriate contraception.

\section{Patients' preferences}

The prerequisites for a profound treatment decision by the patient (and the neurologist) are knowledge of the disease, the treatment options, the efficacy of treatments, potential sides effects, and risks of the planned intervention. MS affects mainly young, often well informed adults, but there are numerous patients who have little insight into the disease and the available therapeutic armamentarium. Obtaining information regarding the patient's knowledge of the disease is therefore important for counseling. Patients and/or their caregivers have to be informed to a point at which decision making is possible. Treatment decisions in MS are becoming increasingly complex. Nowadays, assuring that patients understand the complex disease and the therapeutic field is challenging and also time consuming. 
A shared decision-making process is increasingly advocated as an ideal model of treatment decisions in medical encounters. ${ }^{24}$ In these situations, it is important to notice that patients and clinicians differ in their assessments of the relative importance of different elements of health-related quality of life. ${ }^{25}$

The question of how patient education might influence treatment decisions has been addressed in a 12-month, multicenter, double-blind, randomized, controlled clinical trial in Germany with 192 patients with a diagnosis of RR MS or clinically isolated syndrome. A 4-hour interactive evidence-based educational program was compared with a 4-hours MS-specific stress management program. The primary endpoint was the proportion of participants displaying "informed choice" after 6 months of follow-up, comprising risk knowledge and congruency between attitude towards immunotherapy and actual immunotherapy uptake. Fifty of $85(59 \%)$ participants in the intervention group achieved informed choice after 6 months compared with 18 of $89(20 \%)$ in the control group $(P<0.001)$. The intervention group showed good risk knowledge (significant difference between groups, $P<0.001$ ). There were trends towards increased autonomy preference after the intervention and increased adherence to immunotherapies. ${ }^{26}$

Factors influencing the choice of treatment in clinical practice arise from the neurologist's and the patient's perspective. In a survey of 102 neurologists, the most important attributes of DMT selection were, in order of importance, efficacy, safety, tolerability, patient preference, and convenience, ${ }^{27}$ indicating that patient preference was regarded as a less-important factor. According to Heesen et al however, $80 \%$ of MS patients demand autonomous roles in treatment decisions, with a higher risk knowledge correlating with a preference for higher autonomy. ${ }^{28}$

A study by Mendel et al investigated whether physicians' recommendations can pull patients away from their preferred treatment options. Inpatients, among them 101 with MS, were presented with a hypothetical scenario: the choice between two drugs. They were first asked about their preferences concerning the two drugs and then they received a (fictitious) clinician's recommendation that was contrary to their preferences. Subsequently, they made a final choice between the two drugs. In the decision scenario, $26 \%$ of the patients with MS followed the advice of their physician and thus chose the treatment option that went against their initial preferences. Patients who followed their physician's advice were less satisfied with their decision than patients not following their physician's advice (for MS, $P=0.009$ ). ${ }^{29}$
Since the waiver of the initial preference may have an impact on adherence, this study highlights the importance of patients being encouraged to establish their preference and decide on a treatment most suitable to them.

With regards to patients, factors influencing treatment decisions have been investigated in few studies. Concerning the question of whether to start or not to start a DMT, the patient's risk attitude could be shown to be of relevance. More risk-seeking patients were less likely to choose a treatment compared with more risk-averse patients $(P<0.01) .{ }^{30} \mathrm{In}$ a survey on the use of disease-modifying drugs in a group of 1,572 patients with MS in the Netherlands, the most important reasons not to start a treatment for MS were the severity and phase of the disease, possible side effects, the advice of the neurologist, and uncertainty about the effect of DMTs. According to the opinion of the patients, the neurologist had the most decisive role in starting or changing DMTs, whilst the patient was primarily responsible in deciding to stop the DMT. ${ }^{31}$

To better understand patients' preferences on DMTs, a choice-based conjoint analysis has been used to estimate the maximum acceptable risk trade-offs for various (hypothetical) DMT benefits. Severe side effect risks had the biggest impact on patient preference, with a $1 \%$ risk decreasing patient preference fivefold compared to no risk (odds ratio $[\mathrm{OR}]=0.22, P<0.001)$. Symptom improvement was the most preferred benefit ( $\mathrm{OR}=3.68, P<0.001$ ), followed by prevention of progression within 10 years $(\mathrm{OR}=2.4, P<0.001)$. In this study, daily oral administration had the third highest DMT preference rating $(\mathrm{OR}=2.08, P<0.001){ }^{32}$ In the study by Utz et al an oral medication was preferred over injections by $93 \%$ of patients, when treatment frequency and frequency of side effects were held constant. However, patients preferred injections when pills had to be taken tid and injections only once per week. Injections were also preferred when pills were associated with frequent side effects. ${ }^{33}$

\section{Patient preferences and teriflunomide}

Teriflunomide is an oral treatment given once daily. Different factors may influence patients' preference (Table 2). The oral application is preferable for patients with injection anxiety refusing an injectable DMT, or who have stopped treatment for injection-related reasons. Compared to a bid or tid medication, patients may prefer the once-daily application of teriflunomide; although, according to our experience, this is crucial only in the minority of patients. Frequent liver enzyme controls within the first half year may be undesired by the patient, but are generally feasible in daily practice. 
Table 2 Teriflunomide: factors potentially influencing patient preference in MS

\begin{tabular}{ll}
\hline Efficacy & $\begin{array}{l}\text { Comparable to interferon beta- Ia sc tiw in } \\
\text { the TENERE study }\end{array}$ \\
Route of administration & Oral \\
Dosing frequency & Once daily \\
Half-life & 19 days (median) \\
Pharmacovigilance & Initially frequent liver enzyme controls \\
& (every 2 weeks) \\
Side effects (selection & Arterial hypertension \\
only) & Gastrointestinal disorders (diarrhea, nausea) \\
& Hair thinning \\
& Hematological abnormalities (leucopenia, \\
& lymphopenia) \\
& Liver enzyme increase \\
\hline
\end{tabular}

Abbreviations: MS, multiple sclerosis; sc, subcutaneous; TENERE, TErifluNomidE and REbif. ${ }^{\oplus}$; tiw, three times weekly.

Teriflunomide may cause gastrointestinal side effects, but in contrast to DMF (which has gastrointestinal side effects that are much more prevalent when taken while fasting), the tolerability of teriflunomide is generally not dependent on the fasting status, resulting in more flexibility of the patient. This is favorable for patients with an irregular routine of the day. Potential side effects of DMTs are a major issue in patient counseling before treatment initiation. In the TENERE trial, the only head-to-head Phase III trial, teriflunomide 7 and $14 \mathrm{mg}$ were compared with interferon beta-1a sc tiw. ${ }^{6}$ Based on the findings of this trial, potential patient preferences in clinical practice between teriflunomide and beta interferons may be discussed. Common adverse events ( $\geq 10 \%$ in any group) reported more frequently with teriflunomide included nasopharyngitis, diarrhea, hair thinning, paresthesia, and back pain. Influenza-like symptoms, Alanine Aminotransferase (ALT) increases, and headache occurred more frequently with interferon beta- $1 \mathrm{a}$. Influenzalike symptoms occurred in $<5 \%$ of teriflunomide-treated patients, but in $>50 \%$ of interferon beta-1a treated patients. These data favor teriflunomide for patients who fear flu-like symptoms, have experienced increasing fatigue or spasticity, or have experienced worsening of other MS symptoms associated with flu-like symptoms while injecting beta interferons. Since the incidence of infections was similar in the IFN $\beta$ - $1 \mathrm{a}$ and teriflunomide $14 \mathrm{mg}$ group, a higher incidence could be observed in the teriflunomide $7 \mathrm{mg}$ group, the fear of infections may have no influence on the patient's treatment decision. Gastrointestinal events, especially nausea and diarrhea, occurred more often (for diarrhea approximately threefold) in teriflunomide- than interferon beta-1a-treated patients. Patients with known gastrointestinal disorders may therefore not prefer teriflunomide. Remarkably, a history of gastrointestinal side effects did not have an impact on gastrointestinal tolerability in DMF-treated patients. ${ }^{34}$ Whether this is also true for teriflunomide has yet to be determined. Patients wishing to avoid gastrointestinal side effects at best will decide for a parenteral therapy. Hair thinning $(5.5 \%$ in the $7 \mathrm{mg}$ and $20 \%$ in the $14 \mathrm{mg}$ teriflunomide group) was much more common with teriflunomide, with the majority of cases occuring during the first 6 months. However only 2.7\% of patients in the teriflunomide $14 \mathrm{mg}$ group discontinued due to hair thinning (no patients in the $7 \mathrm{mg}$ group discontinued due to hair thinning), with most patients recovering while continuing treatment. Since hair thinning is an undesirable side effect for many patients, counseling on the temporary nature before and also during treatment is important. Nevertheless, according to our experience, this potential side effects prompts some (more female than male patients) to decide against teriflunomide.

Since teriflunomide can cause an increase of the systolic and diastolic blood pressure, patients with uncontrolled hypertension will decide against teriflunomide. Although rarely, teriflunomide may induce neuropathies; ${ }^{35}$ a preexisting peripheral neuropathy may therefore be an argument against its use and if used in such cases, regular controls will be necessary.

Mean scores in the Treatment Satisfaction Questionnaire for Medication domains of global satisfaction, side-effects, and convenience were significantly improved with both doses of teriflunomide compared with interferon beta-1a in the TENERE trial. Scores in the effectiveness domain did not significantly differ between teriflunomide $14 \mathrm{mg}$ and interferon beta-1a, but were lower with teriflunomide $7 \mathrm{mg} .{ }^{6}$ These findings, however, may be more relevant for patients' adherence than preference.

In animal studies, teriffunomide has been shown to be selectively teratogenic and embryolethal when administered during pregnancy, even at doses less than those used clinically. Therefore, women planning to become pregnant have to stop teriflunomide; due to the long half-life, the washout procedure described above is necessary. Women with a foreseeable plan to become pregnant should therefore, in our opinion, decide on an alternative therapeutic option, if available.

\section{MS and adherence}

The World Health Organization defines adherence as "the extent to which a person's behavior corresponds with agreed recommendations from a healthcare provider" and stresses the importance of good adherence in achieving the full beneficial 
effects of long-term treatments. ${ }^{36}$ It has been reported that $13 \%-72 \%$ of patients do not adhere to disease-modifying MS treatments, ${ }^{37-41}$ and poor adherence or treatment gaps are associated with a higher rate of relapse. ${ }^{41-46}$

Poor adherence is often caused by side effects resulting in a negative impact on quality of life. ${ }^{47}$ It is therefore imperative to inform patients in detail about the planned therapeutic intervention, its risks, and side effects. Inquiry regarding potential side effects should be made at each visit, especially in active disease, when poor adherence may be suspected.

For oral DMTs, there is only very limited data comparing adherence with injectable DMTs. In a retrospective US study using pharmacy claims of patients who initiated DMTs between October 2010 and February 2011, patients initiating fingolimod $(n=248)$ had the highest average proportion of days covered and medication possession ratio in both experienced and naive DMT patients compared to patients using injectable DMTs $(n=1,643)$. The proportion of patients discontinuing their treatment within 12 months was significantly lower for fingolimod. Adjusted results found that patients self-injecting DMTs discontinued their treatment significantly sooner than fingolimod users. ${ }^{48}$ Another retrospective study based on the PharMetrics Plus ${ }^{\mathrm{TM}}$ US administrative claims database (between October 2010 and September 2011) included patients naive to the index DMT. In this analysis, 3,750 patients (fingolimod $n=889$; glatiramer acetate $n=1,233$; any interferon beta $n=1,341$; natalizumab $n=287$ ) were included. Discontinuation rates (fingolimod 27.9\%; glatiramer acetate 39.5\%; interferon beta 43.7\%; natalizumab 39.5\%) and risk of discontinuation were significantly higher for patients receiving other DMTs compared with fingolimod. Adherence with fingolimod was higher than with the other treatment groups. ${ }^{49}$

It could be shown that in injectable DMTs, only one-third of the reasons found to be responsible for poor adherence are dependent on the application form. ${ }^{39}$ This means that non-injection related factors of nonadherence play a major role, as is known from other chronic disorders like epilepsy or hypertension.

\section{Teriflunomide and adherence}

Teriflunomide is distributed in a blister package, divided in 4 weeks' doses, and labeled with the days of the week, which improves the control over the tablets already taken. The fact that teriflunomide is given once daily may favor adherence since compliance is inversely related to the number of daily doses. ${ }^{50,51}$ Due to the long half-life of teriflunomide, it may be speculated that incomplete adherence does not affect treatment efficacy. However, firstly, there are no data on the effect of poor adherence on teriflunomide efficacy; and secondly, the lower dosage of $7 \mathrm{mg}$ daily has been shown to be less effective than $14 \mathrm{mg}$, indicating that dosage matters. Therefore, patients should, as with other DMTs, be advised to take the medication regularly.

In the TEMSO trial, ${ }^{4} 29 \%$ in the placebo, $25 \%$ in the $7 \mathrm{mg}$, and $27 \%$ in the $14 \mathrm{mg}$ group discontinued treatment. The most common reason was side effects in $28 \%, 41 \%$, and $40 \%$ of discontinuing patients, respectively.

Comparably, in the DEFINE trial ${ }^{52}$ on DMF in RR MS, treatment discontinuation was observed in $35 \%$ of placeboand $31 \%$ of DMF-bid-treated patients. Fifteen percent of placebo-treated patients and $48 \%$ of patients in the DMF cohort stopped medication for side effects.

Although data on treatment discontinuation in clinical trials may not be transferred to adherence in daily clinical practice and robust real-world data are lacking, it is important that patients have realistic expectations of potential side effects and that strategies to monitor and, if possible, manage them are discussed in detail.

\section{Conclusion}

The armamentarium of DMTs in MS is increasingly growing. Treatment decisions are made based upon several factors like disease activity, contraindications to the drugs, and, last but not least, physicians' and patients' preferences. Overall, teriflunomide has demonstrated efficacy and safety in a number of Phase III trials in the treatment of relapsing MS. The convenience of administration, the easy safety monitoring in the long-term course, and the tolerability of teriflunomide make it an attractive agent to add to the currently available treatment of relapsing MS. Characteristics of the drug have an impact on patients' preferences. Due to its oral formulation, patients with fear of injections will decide for teriflunomide or the other approved oral options: DMF or fingolimod (if indicated according to the national label). Among oral treatments, factors favoring teriflunomide compared to DMF may be the once-daily dosing frequency and the lack of necessity to take the drug while nonfasting, which provides more flexibility to the patient's everyday life. The long half-life of teriflunomide, however, may dissuade patients, especially women with a foreseeable wish to become pregnant, from taking teriflunomide. Adherence data in clinical daily practice are lacking for teriflunomide, but based on the experiences with other oral treatments in chronic disorders, it will be a challenging topic. In the currently growing field of MS therapeutics, patient counseling 
and perceiving patients' attitudes will be of tremendous importance for treatment selection and adherence aiming at clinical stability and optimal quality of life.

\section{Disclosure}

Antonios Bayas has received honoraria for serving on advisory boards and as a speaker from Merck Serono, Biogen Idec, Bayer Vital, Novartis and Sanofi/Genzyme; for advisory board activities from TEVA; for trial activities from Biogen Idec, Merck Serono and Novartis; and received grants for congress trips and participation from Sanofi/Genzyme, Biogen Idec and Merck Serono.

Mathias Mäurer recieved honoraria for lectures from Biogen Idec, Novartis, Genzyme, Merck Serono, Bayer Vital, Böhringer Ingelheim, TEVA.

\section{References}

1. Gold R, Wolinsky JS. Pathophysiology of multiple sclerosis and the place of teriflunomide. Acta Neurologica Scandinavica. 2011;124(2):75-84.

2. http://www.ema.europa.eu/docs/en_GB/document_library/EPAR_-Product_Information/human/002514/WC500148682.pdf. Aubagio, Summary of Product Characteristics. Accessed 26.12.2014.

3. Tallantyre E, Evangelou N, Constantinescu CS. Spotlight on teriflunomide. International MS Journal/MS Forum. 2008;15(2):62-68.

4. O'Connor P, Wolinsky JS, Confavreux C, et al. Randomized trial of oral teriflunomide for relapsing multiple sclerosis. The New England Journal of Medicine. 2011;365(14):1293-1303.

5. Confavreux C, O'Connor P, Comi G, et al. Oral teriflunomide for patients with relapsing multiple sclerosis (TOWER): a randomised, double-blind, placebo-controlled, phase 3 trial. The Lancet Neurology. 2014;13(3):247-256.

6. Vermersch P, Czlonkowska A, Grimaldi LM, et al. Teriflunomide versus subcutaneous interferon beta-1a in patients with relapsing multiple sclerosis: a randomised, controlled phase 3 trial. Multiple Sclerosis. 2014;20(6):705-716.

7. Miller AE, Wolinsky JS, Kappos L, et al. Oral teriflunomide for patients with a first clinical episode suggestive of multiple sclerosis (TOPIC): a randomised, double-blind, placebo-controlled, phase 3 trial. The Lancet Neurology. 2014;13(10):977-986.

8. Fukushima R, Kanamori S, Hirashiba M, et al. Teratogenicity study of the dihydroorotate-dehydrogenase inhibitor and protein tyrosine kinase inhibitor Leflunomide in mice. Reproductive Toxicology. 2007;24(3-4):310-316.

9. Kieseier B, Benamor M. Pregnancy Outcomes Following Maternal and Paternal Exposure to Teriflunomide During Treatment for Relapsing-Remitting Multiple Sclerosis. Neurology and Therapy. 2014; 3:133-138.

10. Bar-Or A, Freedman MS, Kremenchutzky M, et al. Teriflunomide effect on immune response to influenza vaccine in patients with multiple sclerosis. Neurology. 2013;81(6):552-558.

11. Polman CH, Reingold SC, Banwell B, et al. Diagnostic criteria for multiple sclerosis: 2010 revisions to the McDonald criteria. Annals of Neurology. 2011;69(2):292-302.

12. National Multiple Sclerosis Society NY. Medications [webpage on the Internet]: Consensus on Disease-Modifying Therapies. http://www. nationalmssociety.org/Treating-MS/Medications. 2014;1-33. Accessed 18.10.2014.

13. Gold R. Diagnosis and treatment in multiple sclerosis. Guidelines of the German Society for Neurology (German). 2014. Accessed 26.12.2014.
14. Heesen C, Kleiter I, Nguyen F, et al. Risk perception in natalizumabtreated multiple sclerosis patients and their neurologists. Multiple Sclerosis. 2010;16(12):1507-1512.

15. http://www.ema.europa.eu/docs/en_GB/document_library/EPAR_Product_Information/human/003718/WC500150521.pdf. Lemtrada, Summary of Product Characteristics. Accessed 26.12.2014.

16. http://www.ema.europa.eu/docs/en_GB/document_library/EPAR_Product_Information/human/000102/WC500029425.pdf. Avonex, Summary of Product Characteristics. Accessed 26.12.2014.

17. http://www.ema.europa.eu/docs/en_GB/document_library/EPAR_Product_Information/human/000136/WC500048681.pdf. Rebif, Summary of Product Characteristics. Accessed 26.12.2014.

18. http://www.ema.europa.eu/docs/en_GB/document_library/EPAR_Product_Information/human/000081/WC500053225.pdf. Betaferon, Summary of Product Characteristics. Accessed 26.12.2014.

19. http://www.ema.europa.eu/docs/en_GB/document_library/EPAR_Product_Information/human/000933/WC500034701.pdf. Extavia, Summary of Product Characteristics. Accessed 26.12.2014.

20. http://www.ema.europa.eu/docs/en_GB/document_library/EPAR_Public_assessment_report/human/002827/WC500170303.pdf. Plegridy, Assessment report. Accessed 26.12.2014.

21. https:/http://www.copaxone.com/Resources/pdfs/PrescribingInformation. pdf. Copaxone, Summary of Product Characteristics. Accessed 26.12.2014.

22. http://www.ema.europa.eu/docs/en_GB/document_library/EPAR_Product_Information/human/002202/WC500104528.pdf. Gilenya, Summary of Product Characteristics. Accessed 26.12.2014.

23. http://www.ema.europa.eu/docs/en_GB/document_library/EPAR_Product_Information/human/000603/WC500044686.pdf. Tysabri, Summary of Product Characteristics. Accessed 26.12.2014.

24. Charles C, Gafni A, Whelan T. Shared decision-making in the medical encounter: what does it mean? (or it takes at least two to tango). Social Science and Medicine. 1997;44(5):681-692.

25. Rothwell PM, McDowell Z, Wong CK, Dorman PJ. Doctors and patients don't agree: cross sectional study of patients' and doctors' perceptions and assessments of disability in multiple sclerosis. $B M J$. 1997;314(7094):1580-1583.

26. Kopke S, Kern S, Ziemssen T, et al. Evidence-based patient information programme in early multiple sclerosis: a randomised controlled trial. Journal of Nneurology, Neurosurgery, and Psychiatry. 2014; 85(4):411-418.

27. HansonKA,AgashivalaN, WyrwichKW, RaimundoK,KimE,BrandesDW. Treatment selection and experience in multiple sclerosis: survey of neurologists. Patient Preference and Adherence. 2014;8:415-422.

28. Heesen C, Kopke S, Richter T, Kasper J. Shared decision making and self-management in multiple sclerosis - a consequence of evidence. Journal of Neurology. 2007;254 Suppl 2:II116-II121.

29. Mendel R, Traut-Mattausch E, Frey D, et al. Do physicians' recommendations pull patients away from their preferred treatment options? Health Expectations: An International Journal of Public Participation in Health care and Health policy. 2012;15(1):23-31.

30. Prosser LA, Kuntz KM, Bar-Or A, Weinstein MC. The relationship between risk attitude and treatment choice in patients with relapsingremitting multiple sclerosis. Medical Decision Making: An International Journal of the Society for Medical Decision Making. 2002; 22(6):506-513.

31. Visser LH, van der Zande A. Reasons patients give to use or not to use immunomodulating agents for multiple sclerosis. European Journal of Neurology: the Official Journal of the European Federation of Neurological Societies. 2011;18(11):1343-1349.

32. Wilson L, Loucks A, Bui C, et al. Patient centered decision making: Use of conjoint analysis to determine risk-benefit trade-offs for preference sensitive treatment choices. Journal of the Neurological Sciences. 2014;344(1-2):80-87.

33. Utz KS, Hoog J, Wentrup A, et al. Patient preferences for diseasemodifying drugs in multiple sclerosis therapy: a choice-based conjoint analysis. Therapeutic Advances in Neurological Disorders. 2014; 7(6):263-275. 
34. Fox EJ, Vasquez A, Grainger W, et al. Gastrointestinal Tolerability of Delayed-Release Dimethyl Fumarate in a Multicenter, Open-Label Study of Patients with Relapsing Multiple Sclerosis. Multiple Sclerosis Journal. 2014;20(S1):205.

35. Leist TP, Freedman SM, Kappos L, et al. Pooled Safety Analyses From the Teriflunomide Clinical Development Program. Multiple Sclerosis Journal. 2014;20(S1):110.

36. Sabaté E, ed. Adherence to Long-Term Therapies: Evidence for Action. World Health Organization, Geneva, Switzerland 2003.

37. Brandes DW, Callender T, Lathi E, O’Leary S. A review of diseasemodifying therapies for MS: maximizing adherence and minimizing adverse events. Current Medical Research and Opinion. 2009;25(1): 77-92.

38. Reynolds MW, Stephen R, Seaman C, Rajagopalan K. Persistence and adherence to disease modifying drugs among patients with multiple sclerosis. Current Medical Research and Opinion. 2010;26(3):663-674.

39. Treadaway K, Cutter G, Salter A, et al. Factors that influence adherence with disease-modifying therapy in MS. Journal of Neurology. 2009;256(4):568-576.

40. Tremlett HL, Oger J. Interrupted therapy: stopping and switching of the beta-interferons prescribed for MS. Neurology. 2003;61(4):551-554.

41. Menzin J, Caon C, Nichols C, White LA, Friedman M, Pill MW. Narrative review of the literature on adherence to disease-modifying therapies among patients with multiple sclerosis. Journal of Managed Care Pharmacy: JMCP. 2013;19(1 Suppl A):S24-S40.

42. Uitdehaag B, Constantinescu C, Cornelisse $P$, et al. Impact of exposure to interferon beta-1a on outcomes in patients with relapsing-remitting multiple sclerosis: exploratory analyses from the PRISMS long-term follow-up study. Therapeutic Advances in Neurological Disorders. 2011;4(1):3-14

43. Al-Sabbagh A, Bennet R, Kozma C, Dickson M, Meletiche D. Medication gaps in disease- modifying therapy for multiple sclerosis are associated with an increased risk of relapse: findings from a national managed care database. Journal of Neurology. 2008;255(Suppl 2):S79.
44. Steinberg SC, Faris RJ, Chang CF, Chan A, Tankersley MA. Impact of adherence to interferons in the treatment of multiple sclerosis: a nonexperimental, retrospective, cohort study. Clinical Drug Investigation. 2010;30(2):89-100.

45. Ivanova JI, Bergman RE, Birnbaum HG, Phillips AL, Stewart M, Meletiche DM. Impact of medication adherence to disease-modifying drugs on severe relapse, and direct and indirect costs among employees with multiple sclerosis in the US. Journal of Medical Economics. 2012; 15(3):601-609.

46. Tan H, Cai Q, Agarwal S, Stephenson JJ, Kamat S. Impact of adherence to disease-modifying therapies on clinical and economic outcomes among patients with multiple sclerosis. Advances in Therapy. 2011; 28(1):51-61.

47. O'Rourke KE, Hutchinson M. Stopping beta-interferon therapy in multiple sclerosis: an analysis of stopping patterns. Multiple Sclerosis. 2005;11(1):46-50.

48. Agashivala N, Wu N, Abouzaid S, et al. Compliance to fingolimod and other disease modifying treatments in multiple sclerosis patients, a retrospective cohort study. BMC Neurology. 2013;13:138.

49. Bergvall N, Petrilla AA, Karkare SU, et al. Persistence with and adherence to fingolimod compared with other disease-modifying therapies for the treatment of multiple sclerosis: a retrospective US claims database analysis. Journal of Medical Economics. 2014;17(10):696-707.

50. Saini SD, Schoenfeld P, Kaulback K, Dubinsky MC. Effect of medication dosing frequency on adherence in chronic diseases. The American Journal of Managed Care. 2009;15(6):e22-e33.

51. Claxton AJ, Cramer J, Pierce C. A systematic review of the associations between dose regimens and medication compliance. Clinical Therapeutics. 2001;23(8):1296-1310.

52. Gold R, Kappos L, Arnold DL, et al. Placebo-controlled phase 3 study of oral BG-12 for relapsing multiple sclerosis. The New England Journal of Medicine. 2012;367(12):1098-1107.
Patient Preference and Adherence

\section{Publish your work in this journal}

Patient Preference and Adherence is an international, peer-reviewed, open access journal that focuses on the growing importance of patient preference and adherence throughout the therapeutic continuum. Patient satisfaction, acceptability, quality of life, compliance, persistence and their role in developing new therapeutic modalities and compounds to optimize

\section{Dovepress}

clinical outcomes for existing disease states are major areas of interest for the journal. This journal has been accepted for indexing on PubMed Central. The manuscript management system is completely online and includes a very quick and fair peer-review system, which is all easy to use. Visit http://www. dovepress.com/testimonials.php to read real quotes from published authors. 